\title{
Invariance Control with Chattering Reduction
}

\author{
Melanie Kimmel and Sandra Hirche \\ Institute for Information-Oriented Control \\ Technische Universität München \\ 80333 Munich, Germany \\ Email: melanie.kimmel@tum.de, hirche@tum.de
}

\begin{abstract}
In many control applications, state and/or output constraints need to be satisfied. For that purpose, artificial limits are imposed using a specially designed control law. Among other methods, invariance control has proven valuable for addressing the problem of state and output constraints in nonlinear control systems. However, invariance controlled systems often exhibit undesirable chattering behavior in particular in digital implementation. In this work, we propose a novel invariance-based control approach, which significantly reduces chattering. We give a condition for stability and restrictions on the admissible constraint configuration. The approach and the results are illustrated in simulations.
\end{abstract}

\section{INTRODUCTION}

In some applications, limits on system states and outputs are desired, for example to impose certain performance specifications, safety margins or to guarantee (practical) stability. One application domain is human-machine interaction with examples in driver assistance, rehabilitation, physical training and assistive robotics in domestic and industrial settings, cf. [1]. This requires a control scheme, which is applicable to nonlinear systems, has real-time capabilities and influences the system behavior only to the extent necessary to ensure adherence to limits. Invariance control is such a control scheme. It provides a straightforward approach for imposing constraints on states and outputs of a nonlinear system. The control mechanism switches between nominal control, whenever constraints are satisfied, and corrective control, when constraint violation is likely. Since a switch to corrective control only occurs on absolute necessity, the system is mostly under nominal control for the desired control task execution.

Model predictive control (MPC) [2] or receding horizon control [3] are alternative, optimization-based approaches, which may, in addition to state and output constraints, also consider input constraints. They are, however, computationally expensive, which renders the application to highdimensional nonlinear systems with real-time requirements difficult. Additionally, the behavior within the admissible set is difficult to predict. As another alternative, the reference governor approach [4] explicitly considers disturbances of the system. However, determining the so-called "safe" sets of the state space requires extensive numerical simulation. The use of Barrier Lyapunov functions allows asymptotical tracking of a reference while bounding all signals, but considers only a single system output [5]. Invariance control, in contrast, is capable of handling multiple outputs and utilizes analytic functions to define admissible configurations.

Invariance control is introduced in [6] for nonlinear, control affine single-input single-output (SISO) systems. A systematic approach of designing the invariance controller as an addition to a nominal controller is introduced in [7]. The invariance control approach is extended to multi-input multi-output (MIMO) systems in [8]. It is well-known that the digital implementation of invariance control may induce high-frequency oscillation at the boundaries [9]. This socalled chattering is caused by the finite sampling time, which delays the switching between nominal and corrective control. Although this effect decreases controller performance and provokes undesired behavior at the constraints, it has received very little attention in the existing literature so far.

In this paper, we present a novel invariance control approach for nonlinear, control affine MIMO systems, which addresses the problem of chattering reduction for an output tracking problem. Based on tools from Lyapunov theory, we provide a condition for stability of the invariance controlled system with chattering reduction. Furthermore, we investigate admissible configurations for the set of constraints. The results presented here generalize and improve our earlier work [9] by allowing systems of arbitrary relative degree. The efficacy of the proposed control approach is demonstrated in simulations on a robotic application example.

The remainder of this paper is organized as follows: Section II gives the necessary background of the control strategy. The novel control scheme is introduced in Section III and a sufficient condition for stability is derived. In Section IV, possible restrictions on boundary definitions are analyzed. The results of a numerical example are presented in Section V. Conclusions are drawn in Section VI.

Notation: Bold small and capital characters are used for vectors and matrices, respectively. Time derivatives of low order are abbreviated by dots $\dot{x}=\frac{\mathrm{d} x}{\mathrm{~d} t}$, derivatives of higher order by $x^{(i)}=\frac{\mathrm{d}^{i} x}{\mathrm{~d} t^{i}}$. The first order Lie-derivative, i.e. the directional derivative of a scalar function $h(\boldsymbol{x})$ in direction $\boldsymbol{f}$ is denoted by

$$
\mathcal{L}_{\boldsymbol{f}} h(\boldsymbol{x})=\frac{\partial h}{\partial \boldsymbol{x}} \boldsymbol{f}
$$

The Lie-derivatives of higher order $\mathcal{L}_{f}^{i} h(\boldsymbol{x})$ are defined recursively. The Euclidean vector norm (2-norm) is denoted by $\|\boldsymbol{x}\|_{2}=\sqrt{\boldsymbol{x}^{\mathrm{T}} \boldsymbol{x}}$ for $\boldsymbol{x} \in \mathbb{R}^{n}$ and the cross product 
of two vectors $\boldsymbol{x}_{1}, \boldsymbol{x}_{2} \in \mathbb{R}^{3}$ is denoted by $\boldsymbol{x}_{1} \times \boldsymbol{x}_{2}$. The expression $\boldsymbol{x}_{1} \preccurlyeq \boldsymbol{x}_{2}$ denotes the element-wise inequality of two vectors $\boldsymbol{x}_{1}, \boldsymbol{x}_{2} \in \mathbb{R}^{n}$. Matrix $\boldsymbol{A}=\left[\boldsymbol{a}_{i}^{\mathrm{T}}\right] \in \mathbb{R}^{k \times n}$ and vector $\boldsymbol{b}=\left[b_{i}\right] \in \mathbb{R}^{k \times 1}$ are derived by stacking the $k$ row vectors $\boldsymbol{a}_{1}^{\mathrm{T}}, \ldots, \boldsymbol{a}_{k}^{\mathrm{T}} \in \mathbb{R}^{1 \times n}$ and scalars $b_{1}, \ldots, b_{k} \in \mathbb{R}$, respectively, in a column

$$
\boldsymbol{A}=\left[\boldsymbol{a}_{i}^{\mathrm{T}}\right]=\left[\begin{array}{c}
\boldsymbol{a}_{1}^{\mathrm{T}} \\
\vdots \\
\boldsymbol{a}_{k}^{\mathrm{T}}
\end{array}\right], \quad \boldsymbol{b}=\left[\boldsymbol{b}_{i}\right]=\left[\begin{array}{c}
b_{1} \\
\vdots \\
b_{k}
\end{array}\right]
$$

\section{INVARIANCE CONTROL}

In this section, a brief overview on invariance control is given and the problem setting is introduced. For a more detailed introduction, the reader is referred to [6]-[8].

Invariance control monitors states and outputs of a system with respect to predefined boundaries. The basic structure of an output tracking problem with invariance control is depicted in Fig. 1. The nominal controller processes the

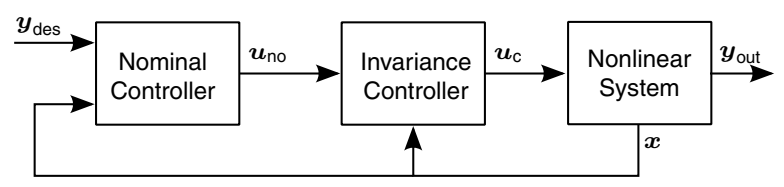

Fig. 1. Structure of an invariance controlled system

output values and determines a control signal. For the invariance control to be stable, the nominally controlled system without invariance control is assumed to be stable [8]. The invariance controller checks the system states, whether a boundary is about to be violated. If this is the case, it emits a corrective control output, which ensures the adherence to the boundaries. Otherwise, the output of the nominal controller is passed through. Therefore, at a distance from the boundaries, the system follows the commands of the nominal controller and whenever it closes in on the constraints, no violation occurs.

In this paper, we consider a nonlinear, control affine MIMO system of the form

$$
\left.\begin{array}{l}
\dot{\boldsymbol{x}}=\boldsymbol{f}(\boldsymbol{x})+\boldsymbol{G}(\boldsymbol{x}) \boldsymbol{u} \\
\boldsymbol{y}_{\text {out }}=\boldsymbol{h}_{\text {out }}(\boldsymbol{x})
\end{array}\right\}
$$

with the state vector $\boldsymbol{x} \in \mathbb{R}^{n}$, sufficiently smooth vector fields $\boldsymbol{f}: \mathbb{R}^{n} \rightarrow \mathbb{R}^{n}, \boldsymbol{g}_{i}: \mathbb{R}^{n} \rightarrow \mathbb{R}^{n}$ with $\boldsymbol{G}=\left[\boldsymbol{g}_{1} \ldots \boldsymbol{g}_{m}\right]$, the input vector $\boldsymbol{u} \in \mathcal{U}_{\text {no }} \subset \mathbb{R}^{m}$, where $\mathcal{U}_{\text {no }}$ is the admissible set for the nominal control output, and the vector of output functions $\boldsymbol{h}_{\text {out }}: \mathbb{R}^{n} \rightarrow \mathbb{R}^{q}$. The nominal control is chosen to ensure a stable system with respect to the tracking error

$$
\boldsymbol{e}=\boldsymbol{y}_{\mathrm{out}}-\boldsymbol{y}_{\mathrm{des}} \text {. }
$$

Assumption 1: There exists a positive definite Lyapunov function $V(\boldsymbol{e})$ for the nominally controlled system $\boldsymbol{u}=\boldsymbol{u}_{\text {no }}$, satisfying

$$
\begin{array}{ll}
\dot{V}(\boldsymbol{e}, \boldsymbol{u}) \leq 0 & \text { for } \boldsymbol{e} \neq \mathbf{0}, \boldsymbol{u}=\boldsymbol{u}_{\mathrm{no}} \in \mathcal{U}_{\mathrm{no}} \\
\dot{V}(\boldsymbol{e}, \boldsymbol{u})=0 & \text { for } \boldsymbol{e}=\mathbf{0}, \boldsymbol{u}=\boldsymbol{u}_{\mathrm{no}} \in \mathcal{U}_{\mathrm{no}}
\end{array}
$$

certifying stability (or with strict inequality $\dot{V}\left(\boldsymbol{e}, \boldsymbol{u}_{\text {no }}\right)<0$ asymptotic stability) in the sense of Lyapunov of the tracking error.

Naturally, the introduced concepts are straightforwardly applicable to systems with single input and/or single output. The constraints on states and outputs are formulated as continuous output functions

$$
y_{i}=h_{i}(\boldsymbol{x}) \leq 0 \quad \text { for } 1 \leq i \leq l,
$$

which equal zero right on the constraint and are negative within the admissible set. These output functions $y_{i}$ are used to derive the invariance control law. There is no upper limit for the number of defined constraints. The entire admissible set $\mathcal{H}$ of the system is given by the set of those state vectors, for which all output functions are non-positive

$$
\mathcal{H}=\left\{\boldsymbol{x} \in \mathbb{R}^{n} \mid \boldsymbol{h}_{i}(\boldsymbol{x}) \leq 0 \quad \forall 1 \leq i \leq l\right\}
$$

Assumption 2: The admissible set is non-empty.

Extending the admissible set, the invariant set takes the system dynamics into account to determine the set to which the states need to be confined. An invariance function $\Phi_{i}\left(\boldsymbol{x}, \gamma_{i}\right)$ of each constraint determines this set. The invariant set of the entire system is the set of state vectors, for which each invariance function takes a non-positive value

$$
\mathcal{G}=\left\{\boldsymbol{x} \in \mathbb{R}^{n} \mid \Phi_{i}\left(\boldsymbol{x}, \gamma_{i}\right) \leq 0 \quad \forall 1 \leq i \leq l\right\}
$$

The boundary $\partial \mathcal{G}$ of the complete invariant set $\mathcal{G}$ is given by all state vectors, for which at least one invariance function equals zero and the others take a non-positive value.

Definition 1: The system (1) is controlled positive invariant, if the controlled system remains within the invariant set (7) for all future times.

We choose the invariance function from [7], which allows the design of the invariance controller as an add-on to a nominal controller. It depends on the relative degree $r_{i}$ of the $i$-th artificial output functions (5), which is determined by input-output linearization

$$
z_{i}=y_{i}^{(r)}=\boldsymbol{a}_{i}^{\mathrm{T}}(\boldsymbol{x}) \boldsymbol{u}+b_{i}(\boldsymbol{x})
$$

$$
\begin{aligned}
\text { with } \boldsymbol{a}_{i}^{\mathrm{T}}(\boldsymbol{x}) & =\left[\mathcal{L}_{\boldsymbol{g}_{1}} \mathcal{L}_{\boldsymbol{f}}^{r_{i}-1} h_{i}(\boldsymbol{x}) \ldots \mathcal{L}_{\boldsymbol{g}_{m}} \mathcal{L}_{\boldsymbol{f}}^{r_{i}-1} h_{i}(\boldsymbol{x})\right], \\
\boldsymbol{a}_{i}^{\mathrm{T}}(\boldsymbol{x}) & =\mathbf{0}^{T} \quad \forall r<r_{i}, \boldsymbol{a}_{i}^{\mathrm{T}}(\boldsymbol{x}) \neq \mathbf{0}^{T} \text { for } r=r_{i}, \\
\text { and } b_{i}(\boldsymbol{x}) & =\mathcal{L}_{\boldsymbol{f}}^{r_{i}} h_{i}(\boldsymbol{x}) .
\end{aligned}
$$

The variable $z_{i}$ is called the pseudo input of the linearized system. If the output functions are chosen such that the systems is not completely linearized, the remaining zerodynamics are assumed to be stable for the controlled system to be stable.

Assumption 3: The system (1) is input-output linearizable with stable zero-dynamics.

The corresponding invariance function to each output function depends on the output function and the respective 
relative degree $r_{i}$ [7]

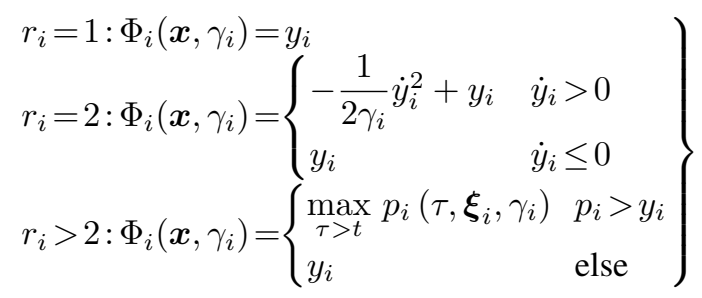

with $\boldsymbol{\xi}_{i}=\left[y_{i} \ldots y_{i}^{\left(r_{i}-1\right)}\right]^{\mathrm{T}}$ and the polynomial

$$
p_{i}\left(t, \boldsymbol{\xi}_{i}, \gamma_{i}\right)=\frac{t^{r_{i}}}{r_{i} !} \gamma_{i}+\sum_{k=0}^{r_{i}-1} \frac{t^{k}}{k !} y_{i}^{(k)} .
$$

The maximization of the polynomial is carried out with respect to future times, i.e. $\tau>t$. The parameter $\gamma_{i}<0$ is a controller parameter. A larger value of $\gamma_{i}$ decreases the time during which corrective control is applied. As shown in [7], the system is made controlled invariant with respect to the invariant set (7), if the invariance function (9) is decreasing on the boundary of the invariant set. As stated there, this is fulfilled, if one of the two conditions

$$
\begin{aligned}
y_{i}^{(r)}(\boldsymbol{x}) & <0 \quad \forall 1 \leq r \leq r_{i}-1 \\
y_{i}^{\left(r_{i}\right)}(\boldsymbol{x}) & \leq \gamma_{i}
\end{aligned}
$$

holds, which is achieved by an invariance control law with a fixed parameter $\gamma_{i}$. Since we concentrate on chattering reduction in this paper, we make the following assumption.

Assumption 4: The original invariance control is designed, such that it renders the system controlled invariant.

A constraint is active and requires a corrective control action if its invariance function $\Phi_{i}\left(\boldsymbol{x}, \gamma_{i}\right)$ has a positive value. If no constraints are active, nominal control is applied. Therefore, only the set of active constraints

$$
\mathcal{K}=\left\{i \in\{1,2, \ldots, l\} \mid \Phi_{i} \geq 0\right\} .
$$

is used for determining the corrective control input. Resulting from (8) and (12), the system is positively invariant with respect to the invariant set (7) of an active constraint $i$, if

$$
z_{i} \leq \gamma_{i}
$$

holds for as long as $\Phi_{i} \geq 0$ [8]. Therefore, the pseudo input is bounded by the control parameter $\gamma_{i}<0$ and a value of $z_{i}=\gamma_{i}<0$ suffices to render the system invariant.

By combining the pseudo inputs (8) corresponding to active constraints with (14), a condition on the control output $\boldsymbol{u}$ is derived [8]

$$
\boldsymbol{A}_{\mathcal{K}}(\boldsymbol{x}) \boldsymbol{u}+\boldsymbol{b}_{\mathcal{K}}(\boldsymbol{x}) \preccurlyeq \gamma .
$$

The condition has to hold element-wise with the matrix $\boldsymbol{A}_{\mathcal{K}}(\boldsymbol{x})=\left[\boldsymbol{a}_{i}^{\mathrm{T}}(\boldsymbol{x})\right]$, the vector $\boldsymbol{b}_{\mathcal{K}}(\boldsymbol{x})=\left[b_{i}(\boldsymbol{x})\right]$, the controller parameter vector $\gamma=\left[\gamma_{i}\right]$ and $i \in \mathcal{K}$.

The corrective control output $\boldsymbol{u}_{\mathrm{c}}$ is determined by solving the constrained minimization problem

$$
\begin{aligned}
\min _{\boldsymbol{u}_{\mathrm{c}}}\left\|\boldsymbol{u}_{\mathrm{c}}-\boldsymbol{u}_{\mathrm{no}}\right\|_{2}^{2} \\
\text { s.t. } \boldsymbol{A}_{\mathcal{K}}(\boldsymbol{x}) \boldsymbol{u}+\boldsymbol{b}_{\mathcal{K}}(\boldsymbol{x}) \preccurlyeq \boldsymbol{\gamma}
\end{aligned}
$$

This ensures that corrective control equals the nominal control $\boldsymbol{u}_{\mathrm{no}}$, whenever no constraint is active and that corrective control is as close as possible to the nominal control in the sense of the Euclidean distance.

Assumption 5: At any instant of time, the number of active constraints (13) is not larger than the dimension of the system input, i.e. $\operatorname{dim}(\boldsymbol{u}) \geq \operatorname{dim}\left(\boldsymbol{b}_{\mathcal{K}}(\boldsymbol{x})\right)$.

Assumption 5 enables an analytical solution of (16)

$$
\boldsymbol{u}_{\mathrm{c}}=\boldsymbol{A}_{\mathcal{K}}^{+}\left(\boldsymbol{z}_{\mathrm{c}}-(\underbrace{\boldsymbol{A}_{\mathcal{K}}(\boldsymbol{x}) \boldsymbol{u}_{\mathrm{no}}+\boldsymbol{b}_{\mathcal{K}}(\boldsymbol{x})}_{\boldsymbol{z}_{\mathrm{no}}})\right)+\boldsymbol{u}_{\mathrm{no}},
$$

where the so-called corrective pseudo input $\boldsymbol{z}_{\mathrm{c}}$ is

$$
z_{\mathrm{c}, i}= \begin{cases}\gamma_{i} & \text { if }\left(z_{\mathrm{no}, i}>\gamma_{i}\right) \wedge\left(\Phi_{i} \geq 0\right) \\ z_{\mathrm{no}, i} & \text { if }\left(z_{\mathrm{no}, i} \leq \gamma_{i}\right) \vee\left(\Phi_{i}<0\right)\end{cases}
$$

and $\boldsymbol{A}_{\mathcal{K}}^{+}=\boldsymbol{A}_{\mathcal{K}}^{\mathrm{T}}\left(\boldsymbol{A}_{\mathcal{K}} \boldsymbol{A}_{\mathcal{K}}^{\mathrm{T}}\right)^{-1}$ is the Moore-Penrose pseudo inverse of $\boldsymbol{A}_{\mathcal{K}}$ [8]. However, due to the finite sampling time in real systems, a system, which is controlled using this approach, shows distinct chattering effects at the boundaries.

\section{Chattering Reduction}

In discrete time implementations of invariance control the invariance function is no longer continuously evaluated in time, but only at the sampling instants. As a result, the switch between nominal and corrective control does not happen immediately, resulting in high-frequency oscillation at the boundaries. This causes a deviation from the desired behavior at the constraints and decreases the controller performance.

\section{A. Adaptation of the Controller Parameter}

In the following, we derive a novel approach for invariance control with chattering reduction. Inspired by [9], the idea is to adapt the controller parameters $\gamma_{i}$ to avoid the overshoots. We consider the $1 \leq i \leq l$ output functions (5) and invariance functions (9) at the $k$-th discrete time step $t_{k}=$ $k \cdot T_{A}$ with the sampling interval $T_{A}$

$$
\begin{aligned}
y_{i, k} & =y_{i}\left(\boldsymbol{x}\left(t_{k}\right)\right) \\
\Phi_{i, k}\left(\boldsymbol{x}, \gamma_{i}\right) & =\Phi_{i}\left(\boldsymbol{x}\left(t_{k}\right), \gamma_{i}\right) .
\end{aligned}
$$

While [9] assumes the relative degree to equal 2, we consider an arbitrary relative degree. We use (9) and (10) to determine the invariance function in the $(k+1)$-th time step. As an example, for a system with relative degree 2 , it evaluates to

$$
\Phi_{i, k+1}\left(\boldsymbol{x}, \gamma_{i}, z_{i}\right)=\left\{\begin{array}{ll}
y_{i, k+1}\left(z_{i}\right) & \dot{y}_{i, k+1}\left(z_{i}\right) \leq 0 \\
y_{i, k+1}\left(z_{i}\right)-\frac{\dot{y}_{i, k+1}\left(z_{i}\right)^{2}}{2 \gamma_{i}} & \dot{y}_{i, k+1}\left(z_{i}\right)>0 .
\end{array} .\right.
$$

The output functions in the following time step depend on the current pseudo control input $z_{i}$. The goal is a non-positive value for the invariance function for the entire sampling interval.

Assumption 6: During one sampling interval, the pseudo control input is constant, i.e. $\boldsymbol{z}(t)=\boldsymbol{z}\left(t_{k}\right) \forall t \in\left[t_{k}, t_{k+1}\right)$. For a system with relative degree $r_{i}$ this means that $y_{i}^{\left(r_{i}-1\right)}$ changes linearly during one time step

$$
y_{i, k+1}^{\left(r_{i}-1\right)}=y_{i, k}^{\left(r_{i}-1\right)}+\int_{t_{k}}^{t_{k}+T_{A}} \underbrace{y_{i}^{\left(r_{i}\right)}}_{=z_{i, k}} \mathrm{~d} t=y_{i, k}^{\left(r_{i}-1\right)}+T_{A} z_{i, k} .
$$


A finite Taylor series determines the values of the lower order derivatives $y_{k+1, i}, \ldots, y_{k+1, i}^{\left(r_{i}-2\right)}$

$$
y_{i, k+1}^{(r)}=\sum_{j=0}^{r_{i}-r} \frac{\left(T_{A}\right)^{j}}{(j) !} y_{i, k}^{(r+j)},
$$

where $y_{i}^{\left(r_{i}\right)}=z_{i, k}$. There are three cases to consider:

1) $\Phi_{i, k}\left(\boldsymbol{x}, \gamma_{i}\right)>0$

2) $\Phi_{i, k}\left(\boldsymbol{x}, \gamma_{i}\right) \leq 0, \Phi_{i, k+1}\left(\boldsymbol{x}, \gamma_{i}, z_{i, k}\right) \geq 0$

3) $\Phi_{i, k}\left(\boldsymbol{x}, \gamma_{i}\right) \leq 0, \Phi_{i, k+1}\left(\boldsymbol{x}, \gamma_{i}, z_{i, k}\right)<0, p_{i}\left(t, \boldsymbol{\xi}_{i, k}, z_{i, k}\right)>0$ for $t_{k}<t<t_{k+1}$ with $p_{i}$ from (10),

where $z_{i, k}$ is the currently applied pseudo control input. In case 1 , the system is not in the invariant set. To enter the invariant set, standard corrective control (18) is applied. In case 2, the application of $z_{i, k}$ leads to a violation of the invariant set in one time step. In 3 , the system is within the invariant set at the current and the following time step. Due to $p_{i}\left(z_{i, k}\right)>0$ for $t_{k}<\tau<t_{k+1}$, however, the invariance is violated in between the sampling instants. Therefore, case 2 and 3 require corrective control.

To minimize the divergence from the nominal system dynamics, the corrective control output is chosen as close to zero as possible, while still being non-positive. The largest, possible value is $z_{\mathrm{c}, i}=0$. Therefore, we distinguish two more cases:

$$
\text { a) } \quad \Phi_{i, k}(\boldsymbol{x}, 0) \leq 0
$$$$
\text { b) } \quad \Phi_{i, k}(\boldsymbol{x}, 0)>0 \text {. }
$$

For case a, the value $z_{\mathrm{c}, i}=0$ suffices to render the system invariant and is used as the corrective output. In case b, a value of $z_{\mathfrak{c}, i}$ is determined, which fulfills the two conditions

$$
\begin{array}{ll}
\text { C1) } & \Phi_{i, k}\left(\boldsymbol{x}, z_{\mathrm{c}, i}\right) \leq 0, \\
\text { C2) } & \Phi_{i, k+1}\left(\boldsymbol{x}, 0, z_{\mathrm{c}, i}\right) \leq 0 .
\end{array}
$$

Condition $\mathrm{C} 1$ ensures the invariance of the system, where

$$
\Phi_{i, k}\left(\boldsymbol{x}, z_{\mathrm{c} 1, i}\right)=0
$$

determines the largest value $z_{\mathrm{c}, i}=z_{\mathrm{c} 1, i}$, which guarantees invariance. Condition $\mathrm{C} 2$ ensures the shortest possible corrective intervention. At the next time step, $z_{\mathrm{c}, i}=0$ suffices to render the system invariant, i.e. $z_{\mathrm{c}, i}<0$ is applied only for one time step. We choose the largest value of $z_{\mathrm{c}, i}=z_{\mathrm{c} 2, i}$, which fulfills condition $\mathrm{C} 2$, to minimize the divergence

$$
z_{\mathrm{c} 2, i}=\max _{\mathrm{C} 2}\left(z_{\mathrm{c}, i}\right) .
$$

In order to fulfill both conditions $\mathrm{C} 1$ and $\mathrm{C} 2$, the minimal value from (24) and (25) is used for corrective control. The final law for the corrective control input is given by

$$
z_{\mathrm{c}, i}= \begin{cases}\min \left(\gamma_{i}, z_{\mathrm{no}, i}\right) & \text { case } 1 \\ \min \left(0, z_{\mathrm{no}, i}\right) & \text { case } 2 \mathrm{a} \vee 3 \mathrm{a} \\ \min \left(z_{\mathrm{c} 1, i}, z_{\mathrm{c} 2, i}, z_{\mathrm{no}, i}\right) & \text { case } 2 \mathrm{~b} \vee 3 \mathrm{~b} \\ z_{\mathrm{no}, i} & \text { else }\end{cases}
$$

with $z_{\mathrm{c} 1, i}$ and $z_{\mathrm{c} 2, i}$ from (24) and (25), respectively. As an example, the corrective pseudo input for a relative degree 2 system is given by

$$
z_{\mathrm{c}, i}= \begin{cases}\min \left(\gamma_{i}, z_{\mathrm{no}, i}\right) & \text { case } 1 \\ \min \left(0, z_{\mathrm{no}, i}\right) & \text { case } 2 \mathrm{a} \vee 3 \mathrm{a} \\ \min \left(-\frac{\dot{h}_{i, k}}{T_{A}}, \frac{\dot{h}_{i, k}^{2}}{2 h_{i, k}^{2}}, z_{\mathrm{no}, i}\right) & \text { case } 2 \mathrm{~b} \vee 3 \mathrm{~b} \\ z_{\mathrm{no}, i} & \text { else. }\end{cases}
$$

The complete corrective pseudo input and the set of active constraints is determined iteratively. In a first step, the conditions for $\boldsymbol{z}_{k}=\boldsymbol{z}_{\text {no }}$ are checked, the constraints, which require a corrective action, are added to the set of active constraints and $\boldsymbol{u}_{\mathrm{c}}$ is determined using (17) for this set of active constraints. Then we choose $\boldsymbol{z}_{k}=\boldsymbol{A}(\boldsymbol{x}) \boldsymbol{u}_{\mathrm{c}}+\boldsymbol{b}(\boldsymbol{x})$ and repeat the previous steps for the remaining inactive constraints, adding them to the active constraints if necessary. This process is repeated until no new constraints are added to the active set. The set of active constraints (13) is now given by

$$
\mathcal{K}=\left\{\begin{array}{l|l}
i \in\{1, \ldots, l\} & \begin{array}{l}
\exists \text { iteration of } z_{i, k} \text { s.t. } \\
\text { case } 1 \vee 2 \mathrm{a} \vee 3 \mathrm{a} \vee 2 \mathrm{~b} \vee 3 \mathrm{~b}
\end{array}
\end{array}\right\} .
$$

\section{B. Stability of Invariance Control with Chattering Reduction}

The derivation of a sufficient condition for stability is based on Lyapunov methods.

Proposition 1: Let $V(\boldsymbol{e})$ be a Lyapunov function for the nonlinear control affine system (1) with nominal control $\boldsymbol{u}_{\text {no }}$, for which Assumption 1 holds. Let Assumptions 2-6 hold. Further assume that the invariance control law (17) with (26) is employed and the stationary point of the reference trajectory $\boldsymbol{y}_{\text {des }}$ lies within the admissible set $\lim _{t \rightarrow \infty}\left(\boldsymbol{y}_{\text {des }}\right) \in \mathcal{H}$. If the element-wise condition

$$
\frac{\partial V}{\partial \boldsymbol{e}} \frac{\partial \boldsymbol{y}_{\text {out }}}{\partial \boldsymbol{x}} \boldsymbol{G}(\boldsymbol{x}) \boldsymbol{A}^{+}(\boldsymbol{x}) \succcurlyeq 0
$$

holds, then the tracking error is stabilized in the sense of Lyapunov. If (3) holds with strict inequality, then the tracking error is asymptotically stabilized in the sense of Lyapunov.

Proof: Using (1) and (2), condition (3) transforms into

$$
0 \geq \frac{\partial V}{\partial \boldsymbol{e}}\left(\frac{\partial \boldsymbol{y}_{\text {out }}}{\partial \boldsymbol{x}}\left(\boldsymbol{f}(\boldsymbol{x})+\boldsymbol{G}(\boldsymbol{x}) \boldsymbol{u}_{\mathrm{no}}\right)-\dot{\boldsymbol{y}}_{\mathrm{des}}\right) .
$$

If the corrective control output fulfills (30) or the stricter condition

$$
\dot{V}\left(\boldsymbol{e}, \boldsymbol{u}_{\mathrm{c}}\right) \leq \dot{V}\left(\boldsymbol{e}, \boldsymbol{u}_{\mathrm{no}}\right) \leq 0,
$$

the invariance controlled system is (asymptotically) stable in the sense of Lyapunov. This is re-written using (30)

$$
\frac{\partial V}{\partial \boldsymbol{e}} \frac{\partial \boldsymbol{y}_{\mathrm{out}}}{\partial \boldsymbol{x}} \boldsymbol{G}(\boldsymbol{x})\left(\boldsymbol{u}_{\mathrm{c}}-\boldsymbol{u}_{\mathrm{no}}\right) \leq 0 \text {. }
$$

Since Assumption 5 holds, it is possible to use (17)

$$
\frac{\partial V}{\partial \boldsymbol{e}} \frac{\partial \boldsymbol{y}_{\text {out }}}{\partial \boldsymbol{x}} \boldsymbol{G}(\boldsymbol{x}) \boldsymbol{A}^{+}(\boldsymbol{x})\left(\boldsymbol{z}_{\mathrm{c}}-\boldsymbol{z}_{\mathrm{no}}\right) \leq 0 .
$$

Element-wise satisfaction of (29) and a non-positive value of each element of $\boldsymbol{z}_{\mathrm{c}}-\boldsymbol{z}_{\mathrm{no}}$ is sufficient for (32) to hold. Therefore, condition (29) is sufficient for stability, if

$$
z_{\mathrm{c}, i}-z_{\mathrm{no}, i} \leq 0
$$


holds for every possible value of $z_{\mathrm{c}, i}$ in (26).

In the first three cases of (26), $z_{\mathrm{c}, i} \leq z_{\mathrm{no}, i}$ holds, since $z_{\mathrm{c}, i}=\min \left(\cdot, z_{\mathrm{no}, i}\right)$. The left side of the inequation (33) is therefore given by

$$
z_{\mathrm{c}, i}-z_{\mathrm{no}, i} \leq z_{\mathrm{no}, i}-z_{\mathrm{no}, i}=0 .
$$

If the forth case applies, $z_{\mathrm{c}, i}=z_{\mathrm{no}, i}$ is used and

$$
z_{\mathrm{c}, i}-z_{\mathrm{no}, i}=z_{\mathrm{no}, i}-z_{\mathrm{no}, i}=0
$$

holds. Therefore, (33) applies in each case and (29) is sufficient to show stability in the sense of Lyapunov for invariance control with chattering reduction as in (26).

Note: The Lyapunov function $V(\boldsymbol{e})$ gives rise to the interpretation as a common Lyapunov function for the switched system. In consequence, arbitrary switching sequences are allowed.

\section{Analysis of the InVARiance Properties}

The stability of the controlled system does not guarantee adherence to the constraints. It remains to show that the novel control law (17) with (26) also renders the system controlled positive invariant. First, we consider the single constraints.

Proposition 2: Let the nonlinear, control affine system (1) be controlled by the nominal control $\boldsymbol{u}_{\mathrm{no}}$. Let Assumptions $1-3,6$ hold. Further, assume there is a sampling instant $t_{k}=k T_{A}$, at which the system is within the invariant set (7). Then, the pseudo corrective control (26) renders the system controlled positive invariant with respect to the set (7) for all $t \geq t_{k}$.

Proof: The system is within the invariant set (7) at $t_{k}=k T_{A}$, meaning that $\Phi_{i, k}\left(\boldsymbol{x}, \gamma_{i}\right) \leq 0$ holds. We show that during the next time step, the system stays within the invariant set, by considering each possible corrective control output. In case $2 \mathrm{a}$ and $3 \mathrm{a}$, the output $z_{\mathrm{c}, i}=0$ is applied and $\Phi_{i, k}(\boldsymbol{x}, 0) \leq 0$ holds. Since $\gamma_{i}<0$ holds, we receive

$$
\Phi_{i}\left(\boldsymbol{x}, \gamma_{i}\right)<\Phi_{i}(\boldsymbol{x}, 0) \leq 0 .
$$

In case $2 \mathrm{~b}$ and $3 \mathrm{~b}$ and for $z_{\mathrm{c}, i}=z_{\mathrm{no}, i}$, either $z_{\mathrm{c}, i} \geq \gamma_{i}$ or $z_{\mathrm{c}, i}<\gamma_{i}$ is possible. For $z_{\mathrm{c}, i}<\gamma_{i}$, the standard invariance condition (14) is fulfilled, ensuring $\Phi_{i}\left(\boldsymbol{x}, \gamma_{i}\right) \leq 0$. Otherwise, for case $2 b$ and $3 b$, condition $\mathrm{C} 1$ holds and

$$
\Phi_{i}\left(\boldsymbol{x}, \gamma_{i}\right) \leq \Phi_{i}\left(\boldsymbol{x}, z_{\mathrm{c}, i}\right) \leq 0
$$

Nominal control $z_{\mathrm{c}, i}=z_{\mathrm{no}, i}$ is applied if $p_{i}\left(t, \boldsymbol{\xi}_{i}, z_{\mathrm{no}, i}\right) \leq 0$ for $t_{k}<\tau<t_{k+1}$ and $\Phi_{i, k+1}\left(\boldsymbol{x}, \gamma_{i}, z_{\mathrm{no}, i}\right) \leq 0$. Therefore

$$
\Phi_{i}\left(\boldsymbol{x}, \gamma_{i}\right) \leq p_{i}\left(t, \boldsymbol{\xi}_{i}, z_{\mathrm{no}, i}\right) \leq 0
$$

holds for $t_{k}<\tau<t_{k+1}$ and $z_{\mathrm{no}, i} \geq \gamma_{i}$. Since in each case, the output is applied for one time step, $\Phi_{i}\left(\boldsymbol{x}, \gamma_{i}\right)<0$ holds at least for $t_{k} \leq t \leq t_{k+1}$ in all of the cases and $\Phi_{i, k+1}\left(\boldsymbol{x}, \gamma_{i}, z_{\mathrm{c}, i}\right) \leq 0$ is true. By mathematical induction, the reasoning is true for all following time steps and therefore the system is rendered controlled positive invariant with respect to (7) for all $t \geq t_{k}$.

This result shows that the linearized dynamics are rendered controlled positive invariant when (26) is applied. It remains to show that for the actual corrective control input $\boldsymbol{u}_{\mathrm{c}}$ (17) the corrective pseudo inputs are combined such that the nonlinear system (1) is rendered controlled positive invariant. Therefore, examine the set of $p$ linear, active constraints

$$
\boldsymbol{h}(\boldsymbol{x})=\left[\begin{array}{c}
\boldsymbol{n}_{1}^{\mathrm{T}} \boldsymbol{x}+c_{1} \\
\vdots \\
\boldsymbol{n}_{p}^{\mathrm{T}} \boldsymbol{x}+c_{p}
\end{array}\right]
$$

with the parameters $c_{i} \in \mathbb{R}$ and the vectors $\boldsymbol{n}_{i} \in \mathbb{R}^{n}$. Inputoutput linearization (8) of system (1), with respect to the output functions (34) yields matrix $\boldsymbol{A}_{\mathcal{K}}$ and vector $\boldsymbol{b}_{\mathcal{K}}$

$$
\begin{aligned}
\boldsymbol{A}_{\mathcal{K}} & =\left[\begin{array}{lll}
\boldsymbol{n}_{1} & \cdots & \boldsymbol{n}_{p}
\end{array}\right]^{\mathrm{T}} \boldsymbol{A}_{\mathcal{K}, r_{i}}=\boldsymbol{N} \boldsymbol{A}_{\mathcal{K}, r_{i}} \\
\boldsymbol{b}_{\mathcal{K}} & =\boldsymbol{N} \boldsymbol{b}_{\mathcal{K}, r_{i}} .
\end{aligned}
$$

with $\boldsymbol{A}_{\mathcal{K}, 1}=\boldsymbol{G}(\boldsymbol{x}), \boldsymbol{b}_{\mathcal{K}, 1}=\boldsymbol{f}(\boldsymbol{x})$ and

$$
\begin{aligned}
\boldsymbol{A}_{\mathcal{K}, r_{i}} & =\left[\mathcal{L}_{\boldsymbol{g}_{1}} \mathcal{L}_{\boldsymbol{f}}^{r_{i}-2} \boldsymbol{f}(\boldsymbol{x}) \ldots \mathcal{L}_{\boldsymbol{g}_{m}} \mathcal{L}_{\boldsymbol{f}}^{r_{i}-2} \boldsymbol{f}(\boldsymbol{x})\right], \\
\boldsymbol{b}_{\mathcal{K}, r_{i}} & =\mathcal{L}_{\boldsymbol{f}}^{r_{i}-1} \boldsymbol{f}(\boldsymbol{x}) \quad \text { for } r_{i} \geq 2 .
\end{aligned}
$$

Assumption 7: The constraint normals $\boldsymbol{n}_{i}^{\mathrm{T}}$ are linearly independent and $\operatorname{rank}\left(\boldsymbol{A}_{\mathcal{K}}\right)=p$ with $\boldsymbol{A}_{\mathcal{K}}$ from (35).

Proposition 3: Consider the invariance controlled, nonlinear, control affine system (1) with the nominal control $\boldsymbol{u}_{\text {no }}$ and let Assumptions 1-6 hold. Further assume that the set of active constraints $\mathcal{K}(28)$ consists of $p$ linear constraints (34), for which Assumption 7 holds. Then, the corrective control (17) with (26) does not cause constraint violation at any instant of time, if

$$
\boldsymbol{z}_{c}-\boldsymbol{b}_{\mathcal{K}} \preccurlyeq 0
$$

with $\boldsymbol{b}_{\mathcal{K}}$ from (36) holds element-wise.

Proof: For the calculation of the pseudo inverse (38), a full rank of $\boldsymbol{A}_{\mathcal{K}} \boldsymbol{A}_{\mathcal{K}}^{\mathrm{T}}$ is necessary. This is the case, if $\operatorname{rank}\left(\boldsymbol{A}_{\mathcal{K}}\right)=p$ holds, which requires the linear independence of the vectors $\boldsymbol{n}_{i}^{\mathrm{T}}$ of the active constraints

$$
\boldsymbol{A}_{\mathcal{K}}^{+}=\boldsymbol{A}_{\mathcal{K}}^{\mathrm{T}}\left(\boldsymbol{A}_{\mathcal{K}} \boldsymbol{A}_{\mathcal{K}}^{\mathrm{T}}\right)^{-1}=\left[\begin{array}{lll}
\boldsymbol{v}_{1} & \cdots & \boldsymbol{v}_{p}
\end{array}\right] .
$$

Since the constraints in $\mathcal{K}(28)$ are active,

$$
z_{\mathrm{c}, i} \leq 0 \quad \forall i \in \mathcal{K}
$$

holds. With (35), (36), the corrective control (17) transforms into

$$
\boldsymbol{u}_{\mathrm{c}}=\boldsymbol{A}_{\mathcal{K}}^{+}\left(\boldsymbol{z}_{\mathrm{c}}-\boldsymbol{b}\right)+(\underbrace{\boldsymbol{I}-\boldsymbol{A}_{\mathcal{K}}^{+} \boldsymbol{A}_{\mathcal{K}}}_{\overline{\boldsymbol{A}}_{\mathcal{K}}}) \boldsymbol{u}_{\mathrm{no}}
$$

Using (38), corrective control is re-written

$$
\boldsymbol{u}_{\mathrm{c}}=\boldsymbol{v}_{1} \cdot\left(z_{\mathrm{c}, 1}-b_{\mathcal{K}, 1}\right)+\ldots+\boldsymbol{v}_{p} \cdot\left(z_{\mathrm{c}, p}-b_{\mathcal{K}, p}\right)+\overline{\boldsymbol{A}} \boldsymbol{u}_{\mathrm{no}} .
$$

For $p=\operatorname{dim}(\boldsymbol{u}), \boldsymbol{A}_{\mathcal{K}}^{+}=\boldsymbol{A}_{\mathcal{K}}^{-1}$ holds for the pseudo inverse and consequently $\overline{\boldsymbol{A}}_{\mathcal{K}}=\boldsymbol{I}-\boldsymbol{A}_{\mathcal{K}}^{-1} \boldsymbol{A}_{\mathcal{K}}=\mathbf{0}$. Therefore, the corrective control output (41) is a sum of $p$ components for $p=\operatorname{dim}(\boldsymbol{u})$ and a sum of $p+1$ components in the case $p<\operatorname{dim}(\boldsymbol{u})$. The first $p$ summands consist of a column of the (pseudo) inverse, multiplied by a non-positive number. 
For the column vectors $\boldsymbol{v}_{i}$ of $\boldsymbol{A}_{\mathcal{K}}^{+}$and the row vectors $\boldsymbol{a}_{i}^{\mathrm{T}}$ of $\boldsymbol{A}_{\mathcal{K}}$, the relationship

$$
\boldsymbol{a}_{i}^{\mathrm{T}} \boldsymbol{v}_{j}= \begin{cases}1 & i=j \\ 0 & i \neq j\end{cases}
$$

holds due to $\boldsymbol{A}_{\mathcal{K}} \boldsymbol{A}_{\mathcal{K}}^{+}=\boldsymbol{I}_{k}$. The maximum increase of the $i$-th output function in the active set (28) is in direction of $\boldsymbol{a}_{i}$. Hence, the positive scalar product implies that in direction of $\boldsymbol{v}_{i}$, the output function $h_{i}(\boldsymbol{x})$ also increases. The value of zero for $\boldsymbol{a}_{j}^{\mathrm{T}} \boldsymbol{v}_{i}$ with $i \neq j$ indicates that the output functions $h_{j}(\boldsymbol{x})$ in the active set remain unchanged in direction of $\boldsymbol{v}_{i}$. Therefore, each $\boldsymbol{v}_{i}$ is admissible to $p-1$ constraints. To reach constraint admissibility with the $i$-th constraint, the vector has to be multiplied by a non-positive number, which is achieved by condition (37). For $k<$ $\operatorname{dim}(\boldsymbol{u})$, there is additionally the component $\overline{\boldsymbol{A}}_{\mathcal{K}} \boldsymbol{u}_{\text {no }}$. Because of the special form of $\bar{A}_{\mathcal{K}}$ (40),

$$
\boldsymbol{A}_{\mathcal{K}} \overline{\boldsymbol{A}}_{\mathcal{K}} \boldsymbol{u}_{\mathrm{no}}=\boldsymbol{A}_{\mathcal{K}}\left(\boldsymbol{I}-\boldsymbol{A}_{\mathcal{K}}^{+} \boldsymbol{A}_{\mathcal{K}}\right) \boldsymbol{u}_{\mathrm{no}}=\mathbf{0}_{p \times 1}
$$

holds, meaning that $\overline{\boldsymbol{A}}_{\mathcal{K}} \boldsymbol{u}_{\text {no }}$ influences none of the output functions and the vector is compliant with the admissible set. Therefore, with condition (37) fulfilled, all of the $p$ or $p+1$ summands are compliant with the admissible set, which holds also for the sum. Since this relationship is independent from the choice of constraint parameters, the sole necessary condition for compliance is the linear independence of the vectors $\boldsymbol{n}_{i}$ and the capability of the system to generate motion in the required direction, which is ensured by $\operatorname{rank}\left(\boldsymbol{A}_{\mathcal{K}}\right)=p$.

\section{Numerical ExAmPle}

In order to illustrate the findings from the previous sections a simulation is carried out in Matlab/Simulink. We compare the proposed novel method to the standard invariance controller [8] and the chattering reduction method presented in our earlier work [9] for different sets of boundaries.

\section{A. Simulation Setup}

We consider a trajectory following problem for a robotic system. The simulation model is shown in Fig. 2. The system

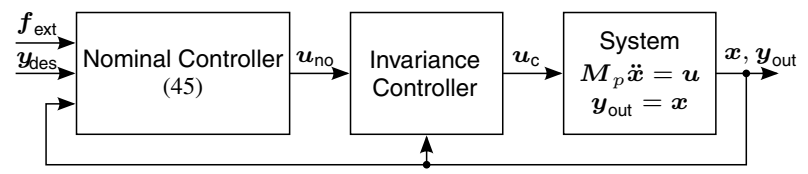

Fig. 2. Structure of the simulation model

is a simplified Cartesian robot model

$$
\boldsymbol{M}_{p} \ddot{\boldsymbol{x}}=\boldsymbol{u}, \quad \boldsymbol{y}_{\text {out }}=\boldsymbol{x}
$$

where $\boldsymbol{M}_{p} \in \mathbb{R}^{3 \times 3}$ is the mass matrix and $\boldsymbol{x} \in \mathbb{R}^{3}$ the state vector consisting of the translational Cartesian directions. The impedance control law [10]

$$
\boldsymbol{u}_{\mathrm{no}}=\boldsymbol{f}_{\text {ext }}+\boldsymbol{D}_{p}\left(\dot{\boldsymbol{y}}_{\text {des }}-\dot{\boldsymbol{y}}_{\text {out }}\right)+\boldsymbol{K}_{p}\left(\boldsymbol{y}_{\text {des }}-\boldsymbol{y}_{\text {out }}\right),
$$

which is used for nominal control, is widely used in robotic applications whenever compliance to exerted forces $f_{\text {ext }} \in$
$\mathbb{R}^{3}$ is desired. For $\boldsymbol{f}_{\text {ext }}=\mathbf{0}$, the control law imitates a spring-damper behavior with the spring constant $\boldsymbol{K}_{p} \in \mathbb{R}^{3 \times 3}$ and the damping constant $\boldsymbol{D}_{p} \in \mathbb{R}^{3 \times 3}$, which enforces the desired motion $\dot{\boldsymbol{y}}_{\text {des }}$. The force input $\boldsymbol{f}_{\text {ext }}$ generates a deviation from the desired motion and ensures compliance. As long as the nominally controlled system is stable, this force does not influence the stability of the invariance controlled system. Then, the external force simply adapts the desired trajectory. The invariance controller ensures that the output $\boldsymbol{y}$ is consistent with predefined constraints. This controller combination allows a compliant reaction to exerted forces within the admissible set and a stiff behavior at the boundaries. The reference trajectory of the system $\boldsymbol{y}_{\text {des }}$ is a single circular movement starting and ending in the same point. No external force is simulated. Three constraints are defined by minimum $x_{1}$ - and $x_{2}$-values and a maximum $x_{1}$ value. The fourth constraint is given by a tilted plane. The simulation is carried out for three different tilted planes. The simulation parameters are listed in Table I.

TABLE I

SimUlATION PARAMETERS

\begin{tabular}{lll}
\hline Sampling time & $T_{A}$ & $0.001 \mathrm{~s}$ \\
Simulation time & $T_{\text {end }}$ & $60.000 \mathrm{~s}$ \\
Cartesian impedance & $\boldsymbol{K}_{p}$ & $600 \mathrm{~N} / \mathrm{m} \cdot \boldsymbol{I}_{3}$ \\
Cartesian damping & $\boldsymbol{D}_{p}$ & $80 \mathrm{~N} \mathrm{~s} / \mathrm{m} \cdot \boldsymbol{I}_{3}$ \\
Mass matrix & $\boldsymbol{M}_{p}$ & $15 \mathrm{~kg} \cdot \boldsymbol{I}_{3}$ \\
External force & $\boldsymbol{f}_{\text {ext }}$ & $\mathbf{0 N}$ \\
Lower position constraints & $x_{1, \min }$ & $0.57 \mathrm{~m}$ \\
& $x_{2, \min }$ & $0.13 \mathrm{~m}$ \\
Upper position constraint & $x_{1, \max }$ & $0.70 \mathrm{~m}$ \\
Tilted plane constraints & $h_{1}$ & $h_{1}=+0.86 x_{1}+x_{2}-1.0 \mathrm{~m}$ \\
& $h_{2}$ & $h_{2}=+0.00 x_{1}+x_{2}-0.4 \mathrm{~m}$ \\
Control parameters & $h_{3}$ & $h_{3}=-2.00 x_{1}+x_{2}+1.0 \mathrm{~m}$ \\
Parameters for [9] & $\gamma$ & -18 \\
& $\alpha_{1}$ & 0.25 \\
& $\alpha_{0}$ & 0.75 \\
\hline
\end{tabular}

\section{B. Results}

The simulation is carried out three times for each control law, once for every set of constraints. The results are shown in Fig. 3. Fig. 3a)-c) depicts the complete trajectory in $x_{1}$ and $x_{2}$-coordinates, the corresponding reference trajectory and the boundaries. In Fig. 3d)-f), the behavior of the system in the right upper corner of the admissible set is shown in more detail. In order to illustrate, that the configuration of the boundaries does not impair the invariance as long as the active constraints are linearly independent, the constraints are chosen such that three significantly different angles occur in the upper right corner.

In Fig. 3a)-c), the trajectories generated by the three control laws almost coincide. The figures show that the boundaries are overall followed by each control law. It can also be observed, that the trajectory starts and ends in the same point in all three cases. This result highlights the stability of the controlled system (Proposition 1).

In Fig. 3d)-f), we observe the differences between the control schemes. The novel control law almost eliminates the chattering effect and the trajectory follows the boundaries 


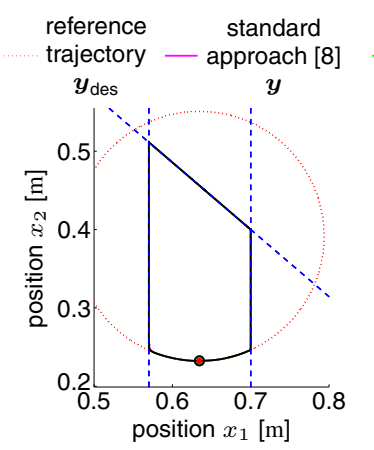

(a)

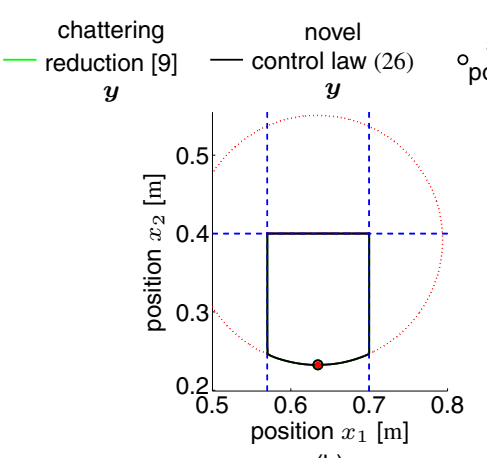

(b)

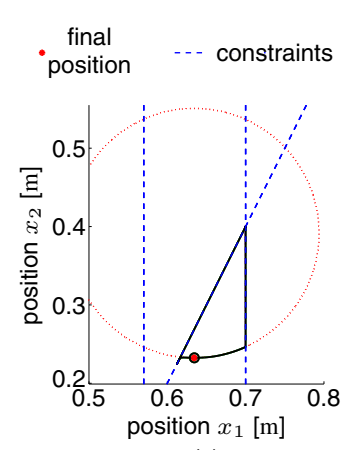

(c)

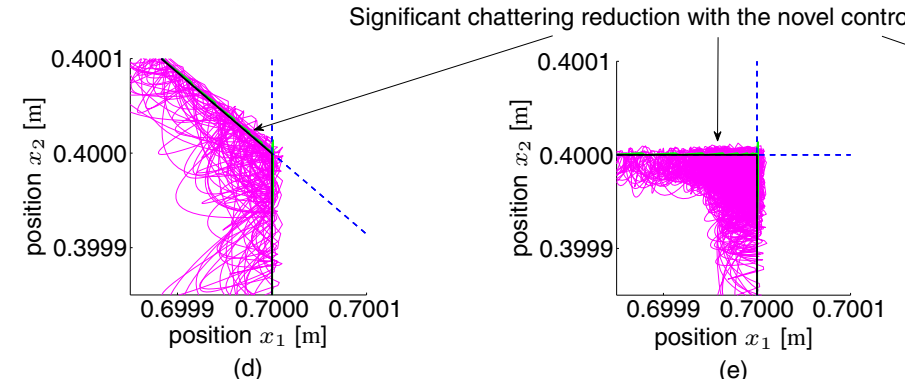

(d)

(e)

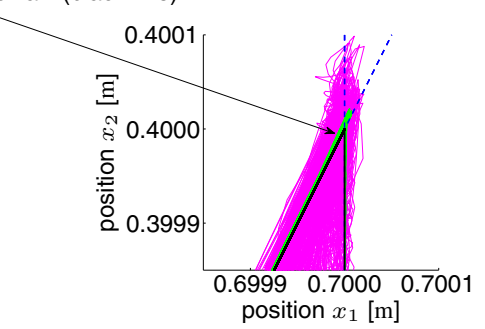

(f)

Fig. 3. Simulation results for three different sets of boundaries: View of the complete trajectory a) with constraint $h_{1}$, b) with constraint $h_{2}$, c) with constraint $h_{3}$; Detailed view of the upper right corner of the admissible area a) with constraint $h_{1}$, b) with constraint $h_{2}$, c) with constraint $h_{3}$

almost exactly. It also shows no violation of the constraints, emphasizing that the system is made controlled invariant (Proposition 2). The standard control scheme and the chattering reduction method from our earlier work, show chattering effects, even despite of the reduction and do slightly violate the constraints. While the standard invariance controller ignores the discrete time implementation of the controller, in our previous work, we use Euler's method to approximate the invariance function in the following time step. Here, we explicitly consider the effects of the discrete implementation of the controller in combination with a continuous system, which yields a more accurate solution and achieves a better controller performance.

\section{CONCLUSION}

In this work, we consider a novel control law for invariance control with chattering reduction. It is analyzed with respect to stability, invariance and applicability with different sets of constraints. We derive a stability condition and additionally, we analyze the controller output with respect to its compliance with the constraints. We show that with linear constraints on the states and/or outputs, the control output renders the system controlled invariant, bounding the states and/or outputs to the admissible set. Simulations illustrate the theoretical results. These findings encourage the use of invariance control a variety of control applications. A remaining challenge is the consideration of time-varying constraints.

\section{ACKNOWLEDGEMENTS}

The research leading to these results has received funding from the European Union Seventh Framework Programme FP7/2007-2013 within the ERC Starting Grant Control based on Human Models (con-humo) under grant agreement no. 337654.

\section{REFERENCES}

[1] J. Medina, M. Lawitzky, A. Mörtl, D. Lee, and S. Hirche, "An Experience-Driven Robotic Assistant Acquiring Human Knowledge to Improve Haptic Cooperation," in IEEE/RSJ International Conference on Intelligent Robots and Systems (IROS), 2011, pp. 2416-2422.

[2] D. Mayne, J. Rawlings, C. Rao, and P. Scokaert, "Constrained model predictive control: Stability and optimality," Automatica, vol. 36, no. 6, pp. 789-814, 2000.

[3] H. Michalska and D. Mayne, "Robust Receding Horizon Control of Constrained Nonlinear Systems," IEEE Transactions on Automatic Control, vol. 38, no. 11, pp. 1623-1633, Nov 1993.

[4] E. Gilbert and I. Kolmanovsky, "Nonlinear tracking control in the presence of state and control constraints: a generalized reference governor," Automatica, vol. 38, no. 12, pp. 2063-2073, 2002.

[5] Z.-L. Tang, K. P. Tee, and W. He, "Tangent Barrier Lyapunov Functions for the Control of Output-Constrained Nonlinear Systems," in 3rd IFAC International Conference on Intelligent Control and Automation Science, P. M. Ferreira, Ed., vol. 3, no. 1, Chengdu, China, 2013, pp. $449-455$.

[6] J. Mareczek, M. Buss, and G. Schmidt, "Sufficient Conditions for Invariance Control of a Class of Nonlinear Systems," in 39th IEEE Conference on Decicion and Control, Sydney, Australia, Dec. 2000, pp. 1436-1442.

[7] J. Wolff and M. Buss, "Invariance Control Design for Nonlinear Control Affine Systems under Hard State Constraints," in NOLCOS'2004 Symposium on Nonlinear Control Systems, Stuttgart, Germany, Sept. 2004, pp. 711-716.

[8] M. Scheint, J. Wolff, and M. Buss, "Invariance Control in Robotic Applications: Trajectory Supervision and Haptic Rendering," in American Control Conference (ACC), Seattle, USA, 2008, pp. 1436-1442.

[9] M. Kimmel, M. Lawitzky, and S. Hirche, "6D Workspace Constraints for Physical Human-Robot Interaction using Invariance Control with Chattering Reduction," in IEEE/RSJ International Conference on Intelligent Robots and Systems (IROS), 2012, pp. 3377-3383.

[10] A. Albu-Schäffer and G. Hirzinger, "Cartesian Impedance Control Techniques for Torque Controlled Light-Weight Robots," in Proceedings of the IEEE International Conference on Robotics and Automation, vol. 1, 2002, pp. 657-663. 